\title{
Epitaxial growth from temporay exhibition to permanent museum in parma
}

\author{
$\underline{\text { Paolo Pio Mazzeo }}^{1}$, Stefano Canossa $^{1}$, Alessia Bacchi ${ }^{1}$, Chiara Massera $^{1}$, Luciano Marchiò ${ }^{1}$, Maria Amarante ${ }^{1}$, Andrea Zappettini $^{2}$, \\ Marina Gorreri ${ }^{1}$ \\ ${ }^{1}$ University Of Parma, Parma, Italy, ${ }^{2}$ IMEM CNR, Parma, Italy \\ E-mail: paolomazzeo.pm@gmail.com
}

The recent International Year of Crystallography held in 2014 spotted the light on a branch of science that significantly shaped our understanding of the world in the last century.

Crystallography allowed scientists to achieve important milestones in the field of structural chemistry/biology with direct implications in medicine, molecular materials, advanced technology and many others. Nevertheless, this fascinating science is often poorly known and barely accessible to the general public even though few important concepts (e.g. double helix of DNA, graphic representation of molecules) are impressed in the collective imagination.

In the last years, we extensively proposed several scientific outreach events addressed to the general public with the aim of showing how crystals are indeed present in our every day life and play an important role in many aspects of our daily activities.

As an example, I would like to present in this context, on behalf of many scientists involved, the exhibition titled "Cristalli!" [1] (trad. "Crystals").

The exhibition spanned different macro areas about crystals-around-us, in particular Crystals in Food, Medicine, Arts, Cultural Heritage, Mineralogy and Technology. Along with the panels, the exhibition also included several pieces of instrumentation, ball-and-stick models of simple crystallographic structures and molecules, and many examples of crystals.

The contents and concepts have been then embedded in a modern Museum, located in the University Science Campus, with a layout especially designed to guide the visitors to appreciate the connections between crystallography and daily life, focused on local specificity such as food excellence and pharmaceutical research.

The exhibition was visited by more than 12000 people in 2014 while at the present the museum is still visited by Primary schools, High schools and by the general public.

In addition, interactive laboratory activities were also proposed both aside the exhibition and as stand-alone projects at different Italian Scientific Festivals[2][3].

This kind of outreach events, mainly addressed to primary schools and general public, consisted in several demonstrative and interactive laboratory activities in which the users

approached the crystallization process by means of simple reactions and consequent characterization of the reaction product.

The IYCr2014 acted as a "seed" for the crystallographic community, extensively supported by IUCr and national associations, to promote a large number of outreach activities, which we hope to continue and let it grow in the future.

[1] http://cristalli.unipr.it/la-mostra.html

[2] http://www.iycr2014.org/countries/events-by-country/italy/crystals-that-change

[3] http://www.iycr2014.org/countries/events-by-country/italy/crystalline-precision

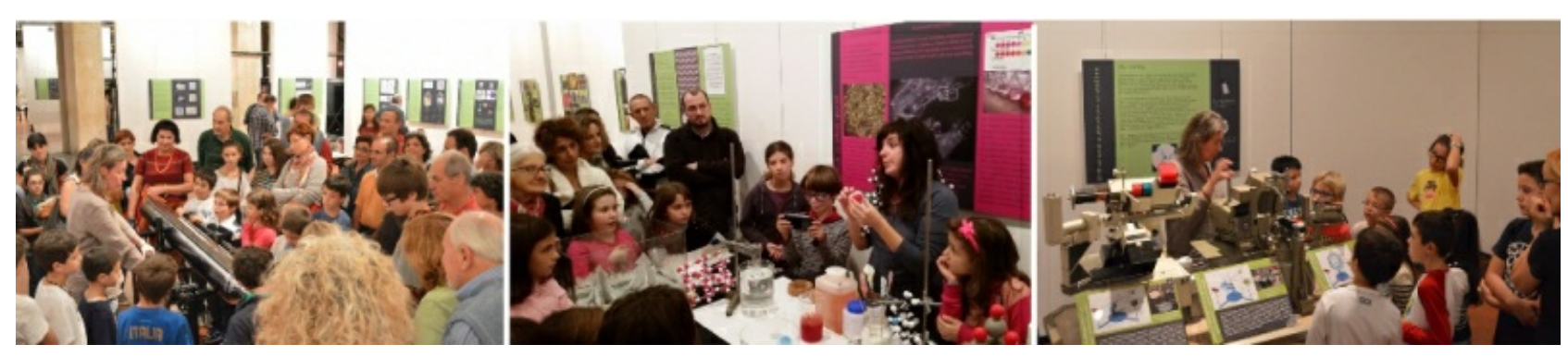

Keywords: Outreach, Exhibition, Interactive Laboratory 